\title{
Pierre Michon : les voix de l'exogenèse
}

\section{Pierre-Marc de Biasi}

\section{(2) OpenEdition}

\section{Journals}

Édition électronique

URL : https://journals.openedition.org/genesis/5683

DOI : 10.4000/genesis.5683

ISSN : 2268-1590

\section{Éditeur :}

Presses universitaires de Paris Sorbonne (PUPS), Société internationale de génétique artistique littéraire et scientifique (SIGALES)

\section{Édition imprimée}

Date de publication : 15 décembre 2020

Pagination : 179-190

ISBN : 979-10-231-0704-3

ISSN : 1167-5101

\section{Référence électronique}

Pierre-Marc de Biasi, «Pierre Michon : les voix de l'exogenèse », Genesis [En ligne], 51 | 2020, mis en ligne le 20 décembre 2021, consulté le 07 février 2022. URL : http://journals.openedition.org/genesis/ 5683 ; DOI : https://doi.org/10.4000/genesis.5683 


\title{
Pierre Michon : les voix de l'exogenèse
}

\author{
Propos recueillis par Pierre-Marc de Biasi
}

Pierre-Marc de Biasi - Pierre Michon, vous avez fait des études de lettres dans les années 1970, au moment où commençait à émerger la notion d'intertextualité : ça doit vous évoquer des souvenirs d'étudiant, non?

Pierre Michon - En fait, pas vraiment... À l'époque, en histoire littéraire à l'Université, je me rappelle qu'on nous parlait plutôt de «sources », d' «inspiration», d' «influence»; il n'était pas beaucoup question d'intertexte dans les discours des profs. Mais ça m'a peut-être échappé : il faut dire que je ne suivais pas non plus très régulièrement les cours. Mais, je me souviens quand même que, de mon côté, je lisais des bouquins de critique, des essais sur la littérature, des revues, etc. où on commençait, par-ci par-là, à entendre parler d'intertextualité. L'exogenèse, c'est venu plus tard...

\section{Mémoire d'intralocution}

P.-M.B. - Pour la génétique, l'exogenèse désigne le processus intertextuel en acte dans l'écriture. Au sens le plus extensif, le phénomène met potentiellement en jeu l'ensemble des auvres littéraires antérieures, aussi bien que n'importe quelle trace écrite se rapportant au monde, aux savoirs, ou même à la vie de l'écrivain, etc.

$P . M$. - Les traces écrites qui se rapportent à ma propre vie? Ah non, là, je ne suis pas d'accord ! C'est bien trop extensif. Non, pour moi, les traces verbales de ma propre vie, ce n'est pas de l'intertextualité. Je pense par exemple à des souvenirs précis d'échanges, de discussions ou d'interlocutions familiales qui ont eu lieu dans ma petite enfance : il y a des phrases entières de mon grand-père ou de ma mère qui se trouvent dans mes livres, mais, quand je les ai écrites, je n'ai pas eu vraiment à les reconstituer, elles sont sorties telles quelles de ma mémoire.

P.-M.B. - Comment définiriez-vous cette spontanéité mémorielle?

$P$. $M$. - En fait il s'agit d'un phénomène qui relève de ce qu'il faudrait appeler non pas de l'interlocution, puisque les locuteurs n'existent plus, mais de l' «intralocution» : ça me parle du dedans, avec leurs voix ; et leurs paroles, je peux les articuler sans remuer les lèvres, comme le ventriloque sait parler avec une voix qui vient du ventre. Ces bribes de discours, en dehors de moi, personne ne les connaît ni ne pourra jamais les connaître. S'il y a «emprunt», son origine est complètement intérieure, invisible et privée. Et, à mon sens, ça n'a rien à voir avec les textes littéraires que je peux connaître par cœur.

P.-M. B. - Pourtant, hormis le fait notable qu'elle n'est pas une trace écrite, mais une intralocution, cette phrase de votre mère ou de votre grand-père, si elle est inscrite et présente dans votre mémoire, ne l'est-elle pas au même titre que l'intertexte littéraire que vous avez intériorisé?

P. M. - Non, il y a une énorme différence. Dans le cas de cette inter- ou intra-locution familiale - qu'il s'agisse des lettres de mon grand-père, ou des paroles de ma mère - ce sont des souvenirs intimes, des traces psychiques qui n'ont aucun autre support que ma mémoire personnelle : selon moi, ça relève strictement de ce que vous appelez l'endogenèse. Pour les textes littéraires que je porte en moi, c'est autre chose : ils gardent un certain coefficient d'extériorité. Là, on peut parler d'intertextualité parce qu'il existe par ailleurs une version écrite de ces textes, une version publique et objective à laquelle n'importe qui peut avoir accès et se reporter, et parce que les phrases qui les composent n'existent pas seulement dans ma mémoire. 


\section{Bibliothèque intérieure}

P.-M. B. - Parlons de tous ces textes littéraires qui vous habitent. Il s'agit donc bien pour vous d'une matière intertextuelle, même si vous n'avez pas besoin d'aller la rechercher dans des livres. Qu'y a-t-il dans cette bibliothèque intérieure? Vous-même, le savez-vous?

P. $M$. - Évidemment, non... Je vois à peu près de quoi il s'agit pour quelques écrivains et quelques œuvres, mais je n'ai aucune idée précise du tout, ni de son périmètre. D'ailleurs je ne sais pas si on peut parler de «bibliothèque »... de florilège, oui. Il y a des textes qui me reviennent, dont je ne savais même pas que je les connaissais par cœur, d'autres que je croyais connaître sur le bout des doigts et qui, par moments, me restent sur le bout de la langue. L'anamnèse est capricieuse. Ça dépend beaucoup des circonstances.

P.-M. B. - Et de quelle manière ces textes agissent-ils sur votre travail? Est-ce par une démarche volontaire de remémoration, ou comme à votre insu?

P. M. - Plutôt sans que je le sache, j'ai l'impression... sans que je le cherche, en tout cas... Ça peut, ça doit venir comme ça, spontanément, dans le mouvement de l'écriture : ça a lieu, c'est tout. Difficile de dire pourquoi.

P.-M. B. - Et quelle forme cela peut prendre? Est-ce une affaire de rythme? d'images? de formules?

P. M. - Les rythmes, oui... la scansion du vers. À beaucoup de moments de mon travail - et d'ailleurs trop souvent, il faudrait que j' arrive à refréner cette tendance - je me sens entrainé par la rythmique de l'alexandrin, la scansion Hugo : ça, c'est un truc qui me hante littéralement.

P.-M.B. - L'alexandrin, mais peut-être pas seulement? Il m'a semblé par exemple, dans Les Onze, qu'il y avait des effets de rythmes différents, proches de ceux d'Antonin Artaud...

P. M. - C'est bien possible! Oui... Ta ta ta ta ta ta ta... ta ta! Le rythme Artaud est octosyllabique. Ce n'est pas l'alexandrin. C'est autre chose, mais c'est puissant. Et ça peut agir aussi fortement sur ce qu'on se formule mentalement en écrivant. Sûrement.

P.-M. B. - Dans Les Onze, j'ai même eu l'impression, çà et là, de reconnaître des mots, des adverbes, qui pourraient provenir du Van Gogh... Si c'est le cas, s'agit-il de clins d'ail?

P. M. - Des expressions d'Artaud ? C'est bien possible aussi, il fait partie de ma bibliothèque intérieure. Mais là, je ne suis pas sûr. Les Onze, c'est beaucoup plus sophistiqué qu'Artaud. Artaud est surtout bon pour la colère, pour ses bouffées d'exaspération incantatoire, pour tous les moments où sa rage explose. Donc, non, je ne crois pas. Et si c'était le cas, il ne s'agirait pas de clins d'œil : ce n'est pas vraiment ma façon de fonctionner. Il y a quand même, de temps en temps, des affaires de clin d'œil dans mon écriture, mais ce sont des exceptions. Pour la plupart des citations, ce sont des hommages, que le lecteur lettré peut reconnaître, mais qui ne sont pas là pour ça. Leur rôle n'est pas de déclencher la lecture intertextuelle, pas du tout. Le premier récit des Vies minuscules est carrément truffé de citations de Chateaubriand, de Hugo, etc. Par exemple - c'est juste un cas qui me revient - quand j'évoque le rivage des Açores où le héros a abordé, le texte dit : «dans les prairies peinturées de papillons et de fleurs...». Eh bien, cette formule pleine d'allitérations en «p», elle vient de Chateaubriand : c'est une citation, mais elle m'est venue comme ça; elle n'est pas là pour faire clin d'œil, pour suspendre le narratif et créer de la distanciation, au contraire ! Elle est là simplement parce que c'est une formule que je trouvais parfaite pour donner à voir ce que j'avais à l'esprit, et c'est pour cela qu'elle est sans guillemets ni italiques.

Le clin d'œil, c'est autre chose. Prenons un autre exemple. Dans le texte que je suis en train de faire en ce moment, le narrateur (qui n'est pas du tout moi) est un écrivain célèbre qui s'y croit, qui se prend un peu pour, mais qui se moque aussi de lui-même sans cesse (finalement si, c'est quand même un peu moi). Or, à un moment où il raconte sa vie, il se la joue carrément en disant : «J'ai fait la magique étude du bonheur que nul n'élude. J'ai fait bien d'autres choses ». D'un côté, il y a l'intertexte, les deux vers de Rimbaud ${ }^{1}$, par

1. Arthur Rimbaud, Derniers Vers, «Bonheur» : «J'ai fait la magique étude / Du Bonheur, que nul n'élude.» 
lesquels le narrateur joue, sans s'en cacher, le rôle convenu de l'écrivain cultivé, et puis le «J'ai fait bien d'autres choses » par lequel il revient à son ego avec une complaisance tout aussi ironique. Au lecteur de s'en amuser ou pas, mais on est bien là dans le cas de figure du clin d'œil.

Dans Les Onze aussi, il y en a plein, mais ce sont des clins d'œil qui n'ont pas été aperçus. Par exemple, il y a des phrases de Michelet texto, enfin des fragments de phrases. À un moment, Michelet parle, je ne sais plus où 2 , de «l'épée enchantée du général Bonaparte». Moi, je parle des représentants en mission, incapables de prendre le pouvoir, en disant : «ils attendaient, le plumet très professionnel, l'épée enchantée du général Bonaparte».

Mais tenez, puisqu'on se demandait tout à l'heure si l'interlocution familiale peut être considérée comme de l'intertexte, parlons du «plumet». Ce fameux «plumet»dont je fais tant usage dans Les Onze, où il est l'insigne même du militaire, de l'héroïsme enchanté, ce plumet vient de ma mère. Comment expliquer cela? Pour moi le plumet signifie l'ébriété. Parce que ma mère disait d'un tel ou d'un tel : «Ah! j'ai vu passer Rossignol tout à l'heure dans la rue... il avait bien son petit plumet!» Ça voulait dire : visiblement, il en avait un bon coup dans le nez, il était ivre mort! Donc, pour moi, quand j'écris le mot «plumet», même le plus sérieusement du monde, je ne peux pas ne pas y penser. Donc il y a l'intertexte de Michelet, mais derrière il y a aussi la parole de ma mère : c'est de l'exogenèse à double fond !

Je pense à une autre citation textuelle de Michelet dans Les Onze. Au moment de l'exécution de Danton et d'Hébert, je dis : «Robespierre sortit le couteau pour raser de droite et de gauche, les modérantins et les exagérés, le beau couteau nommé Saint-Just ${ }^{3}$. » Ça vient directement de Michelet qui avait parlé du «couteau aigu de Saint-Just ${ }^{4}$ ». En fait, il y a beaucoup de Michelet dans Les Onze...

P.-M. B. - Au point que Michelet comparaît en personne dans le texte!

P. M. - Oui ! Michelet est présent physiquement dans le récit; mais ce sont justement les citations de Michelet qui ont suscité l'apparition de son corps dans ma tête...

P.-M. B. - Donc tout s'est passé comme si, invoqué par son énonciation, l'intertexte avait fini par s'incarner, par prendre corps dans la fiction! La voix comme incarnation, l'intertextualité comme résurrectionnisme, ça ne pouvait que faire plaisir à Michelet.

P. M. - C'est ça... Exactement comme vous le dites.

\section{Ambivalences de l'exogenèse}

P.-M. B. - Pour les citations, c'est spontanément que les phrases de Michelet vous venaient à l'esprit au fil de votre rédaction? Ou bien s'agissait-il d'une stratégie à l'échelle du texte tout entier?

P. M. - Oui, plutôt spontanément, mais ça n'empêche pas des arrière-pensées plus globales. Il n'y a pas que du Michelet... Tout le chapitre sur les représentants, les passages sur les mises à mort à Nantes, sur Carrier, c'est comme du Hugo des Soldats de l'an Deux, mais noirci, donc comme du Shakespeare ! Noirci justement parce que la magique noirceur shakespearienne était sur eux. Il y a beaucoup de Shakespeare dans Les Onze : par exemple, il y a un chapitre qui commence par «Où en est la nuit, Monsieur?»... J'adore ce «Où en est la nuit?» Il n'est pas de moi. C'est ce que dit, à son fils, Duncan, le personnage qui accompagne le roi d'Écosse chez Macbeth.

Il y a un autre truc que $\mathrm{j}$ ' aurais voulu placer, mais que je n'ai finalement pas réussi à utiliser, qui est aussi de Michelet. Quand il commence à parler de la Terreur, il dit [Michon prend une voix caverneuse] : «J'entre avec mon sujet dans la nuit et dans l'hiver...» J'avais envie de citer ça parce que la deuxième partie des Onze a été écrite à Nantes et que c'est aussi à Nantes que Michelet a écrit ses pages sur la Terreur...

2. La citation de Michelet provient du texte : Les Soldats de la Révolution, Première partie, Sous la République, «Nos armées républicaines »: «Je connaissais à merveille l'épée enchantée, infaillible, que la Révolution mourante avait placée dans ma main. » Michelet imagine ce que Napoléon aurait dû écrire dans ses Mémoires.

3. Pierre Michon, Les Onze, Paris, Verdier, 2009, p. 18.

4. «Robespierre tournait autour pour percer les fondements. Nous allons le voir encore, pour cette œuvre difficile, employer une arme neuve, le couteau aigu de Saint-Just. », Histoire de la Révolution, IX, 6, «Le Procès. Essai de la gauche pour terroriser le centre et les neutres. Lutte de Cambon et de Robespierre». 
P.-M.B. - Citation géolocalisée : GPS intertextuel! Mais quand vous dites "quelque chose que j'aurais voulu placer...» qu'est-ce que vous voulez dire exactement? Ça désigne quel geste d'écriture? Quelle logique intertextuelle?

P. M. - Ah! C'est vrai, j'ai dit ça... Là, vous m'avez piégé !...

P.-M.B. - Non... je voudrais juste comprendre de quel jeu il s'agit, ce qui préside à ce jeu... Est-ce un hommage? L'affichage d'une connivence?

P. M. - Oui, si je cite, c'est par hommage et par connivence, mais c'est en même temps, presque toujours, par ironie, pour me moquer. C'est une moquerie, envers celui que je cite, envers moi-même et envers le fait même de citer. Je me moque de la vieille machine à parler, de ce truc éculé qu'on appelle la culture, la transmission, la tradition et qui est fondé sur la citation. Parce que dans la transmission, il ne faut jamais oublier le cancre du fond de la classe, le pauvre idiot, le laissé pour compte qui y croit mais qui n'y arrive pas parce qu'il n'a pas les codes. Il ne voit pas de quoi on lui parle, il n'en peut plus de toutes ces citations, il voudrait envoyer tout ça au Diable, ne plus jamais en entendre parler. Et il n'a pas forcément tort. Citer, c'est le geste de l'auctoritas... On peut avoir des raisons de s'en méfier. D'ailleurs avant d'être une complaisance littéraire, la citation est une assignation : c'est la sommation à comparaître devant un tribunal pour être jugé. C'est pour ça qu'avant de s'en servir, il faut prendre garde à la violence, à l'aplomb et à la suffisance inouïe qu'il y a à citer... Bref, mesurer ce que la citation doit à la bêtise, comme dirait Flaubert, à la pire, à celle qui tue.

P.-M. B. - Mais, d'un autre côté, citer peut aussi être perçu à bon droit comme un exercice bienveillant d'admiration qui renvoie à une galerie de grands ancêtres, à un Panthéon personnel.

P. M. - Qui y a-t-il dans mon Panthéon privé? Je ne sais pas... À part Flaubert, qui sert justement à penser, je n'y pense jamais. Je crois qu'il y a beaucoup de monde dans mon Panthéon, mais je sais qu'il y a aussi pas mal de figures taboues : des écrivains que j'admire, mais qui ont un fan-club si pléthorique que j'ai dû les mettre au placard... D'ailleurs même chez Flaubert, je bute sur ce genre d'interdit : c'est très difficile de le citer. Il est victime de son succès. Tout le monde par exemple imite une phrase de Bovary, célèbre par la manière dont elle se termine : virgule, «la nuit», point. $\ll \ldots$ ta ta ta /ta ta, la nuit ${ }^{5}$.» C'est magnifique : en deux mots, il y a toute l'obscurité et toute l'opacité de la nuit la plus noire, sans qu'il soit nécessaire d'ajouter quoi que ce soit au mot «nuit», grâce à cet amuïssement du souffle qui rejette le mot en fin de phrase et clôt le tout sur deux syllabes. C'est parfait, mais inutilisable : cette phrase, je l'ai trouvée mille fois chez nos écrivains cultivés. Même chose pour le «Puis tout disparut ${ }^{6} »$ au début de L'Éducation.

\section{Lambeaux de mémoire textuelle}

P.-M. B. - Les éléments d'exogenèse qui parviennent à s'insérer dans l'œuvre n'apparaissent pas à n'importe quelle phase du travail de l'écriture. Ils ont tendance à se profiler au moment de la rédaction, avec la formation des phrases. Mais ils peuvent aussi induire des transformations à une autre échelle. On l'a vu : citer Michelet peut donner envie de le convoquer dans la fiction. En fait, par quel processus et à quel moment l'écriture s'engage-t-elle dans l'exogenèse?

P. M. - Oui, même s'il m'arrive de glaner des bouts de textes et des citations en amont, quand je prépare un projet dans mes carnets, il est certain que le moment de vérité, pour l'exogenèse, c'est le travail rédactionnel. Comment ça se passe ? Pas facile à dire... Tout à coup la phrase qui s'écrit bute sur une idée, un mot, une image, qui fait surgir une sorte de fantôme intertextuel : un lambeau de mémoire textuelle que l'écriture ramasse et qu'elle transcrit pour voir s'il y a quelque chose à en faire. Après, quand la formule est redéployée, il y a l'étincelle et le coup de foudre, ou bien

5. «On parla d'abord du malade, puis du temps qu'il faisait, des grands froids, des loups qui couraient les champs, la nuit.» (Gustave Flaubert, Madame Bovary, ch. II.)

6. «Un peu plus loin, on découvrit un château, à toit pointu, avec des tourelles carrées. Un parterre de fleurs s'étalait devant sa façade; et des avenues s'enfonçaient, comme des voûtes noires, sous les hauts tilleuls. Il se la figura passant au bord des charmilles. À ce moment, une jeune dame et un jeune homme se montrèrent sur le perron, entre les caisses d'orangers. Puis tout disparut.» (Gustave Flaubert, L'Éducation sentimentale, Première partie, ch. I.) 
l'indifférence et l'abandon. L'écriture en fait sa chose ou renonce à s'en saisir. C'est un processus mental et graphique à la fois. Mais il arrive aussi que ça passe par la médiation des carnets. Pour la phrase de Michelet sur «l'épée enchantée du général Bonaparte », par exemple, je crois bien que je l'avais notée antérieurement. Le calepin est un pense-bête, une mémoire externalisée, une sorte de florilège provisionnel où je compile tout ce qui m'intéresse : avoir consigné un fragment de texte dans un carnet aide à le retrouver plus facilement mais sert aussi à le maintenir actif dans son esprit. Et ce n'est pas tout. Au-delà de son côté pragmatique et utilitaire, comme outil de doc, le carnet est en lui-même un instrument de jouissance, une sorte de réserve d'énergie vitale pour l'écriture : il a partie liée avec ce qui forme le désir et la puissance d'écrire. Cette phrase que j'ai notée, le carnet me permet de la relire et d'en jouir à loisir : le plaisir qu'elle me donne se traduira peut-être par le besoin ou le désir de la placer dans mon texte, mais pas forcément : même si elle n'y trouve pas sa place, elle pourra avoir été à l'origine de l'impulsion qui m'a fait écrire. Ça, c'est une forme secrète et très singulière de l'exogenèse...

P.-M. B. - Dans cet échange entre lecture et écriture, est-ce qu'il vous arrive de jouer vous-même au critique, de débusquer par exemple des cas d'intertextes cachés chez les écrivains que vous lisez?

P. M. - Ça m'arrive; je peux même vous raconter un cas tout récent, à propos de Victor Hugo. Avant-hier, je lisais à ma fille Louise un poème des Contemplations :

Elle était pâle, et pourtant rose,

Petite avec de grands cheveux.

Elle disait souvent : Je n'ose,

Et ne disait jamais : Je veux.

Louise me dit «C'est tout comme moi !», par antiphrase, naturellement... Mais au moment où je lui lisais ces vers de Hugo, il y avait un autre texte qui me revenait à l'esprit : vous savez, ces vers du Roman inachevé d'Aragon, dans le très célèbre poème «Est-ce ainsi que les hommes vivent ${ }^{7}$ » :

Elle était brune et pourtant blanche

Ses cheveux tombaient sur ses hanches

Et la semaine et le dimanche

Elle ouvrait à tous ses bras nus.
Et voilà comment Aragon réécrit Hugo, en inversant son intertexte : d'un côté l'ange, de l'autre la pute... Des jeux intertextuels comme ça, il y en a plein mes textes.

P.-M. B. - Aragon voulait être le Hugo du XXe siècle, il a vécu dans son intertexte sans s'en cacher. Revenons sur la question des degrés dans l'affichage de l'emprunt. Est-ce que vous pratiquez la citation avec guillemets?

$P$. $M$. - Je la pratiquais quand j'étais encore timide. Depuis, il peut m'arriver évidemment d'expliciter le nom de l'auteur en mettant «Comme disait Untel», et en donnant la citation en italiques; quelquefois, je m'amuse à ne rien préciser du tout, et la plupart du temps, l'emprunt est bien là mais reste parfaitement invisible. Par exemple, dans le Watteau, il y a l'expression «l'essaim des feuilles d'or», pour évoquer un parc XVIII' siècle : c'est du Rimbaud! La formule sort directement des Illuminations : «L'essaim des feuilles d'or entoure la maison du général ${ }^{8}$.»

P.-M. B. - Ce genre de geste intertextuel, c'est un plaisir que vous vous faites en écrivant? Ça reste une affaire entre vous et vous, ou bien est-ce l'esquisse d'un dialogue avec le lecteur idéal que vous imaginez?

P. M. - C'est à la fois un plaisir que je me fais et un truc spontané qui vient tout seul. Je ne me dis pas : «Tiens, là, je vais placer cette formule de Rimbaud. » Ça peut m'arriver de chercher à faire sourire mon lecteur, mais en l'occurrence, non, j'étais certain que ça passerait inaperçu... Non, «l'essaim des feuilles d'or» est arrivé dans ma phrase parce que c'était exactement ce qu'il fallait pour le souffle 9 .

7. Louis Aragon, Le Roman inachevé, incipit «Bierstube Magie allemande», Paris, Gallimard, coll. «Poésie», p. 74.

8. Arthur Rimbaud, Illuminations, «Enfance II».

9. Pierre Michon, Maîtres et Serviteurs, «Je veux me divertir», Verdier, 1990 , p. 67 : «dans la plus belle maison de Nogent, un palais à rocailles et fontaines avec un parc encore et des terrasses sous l'essaim des feuilles d'or, la maison d'été de Le Fevre [...]». 


\section{L'intertexte tabou}

P.-M. B. - Dans le projet de rédaction qui vous occupe actuellement, vous disiez que le travail ou le jeu avec l'intertexte jouait un rôle particulièrement important. De quelle manière?

P. M. - Oui, il y a beaucoup de Borges dans mon livre; et il y a aussi - puisqu'on en parlait tout à l'heure - une sorte de volontarisme qui pourrait se rapprocher d'Antonin Artaud : une sorte d'ordre, d'injonction faite à l'ordre du Monde de se conformer à ce que dit le texte : là, c'est vraiment Artaud. Mais le véritable intertexte, celui qui est omniprésent, c'est Borges. Il est partout, surtout par le rythme. Pour ce que je suis en train de faire, c'est une référence presque obligée. Donc, Borges pour la scansion, Artaud pour l'injonction.

P.-M. B. - Et à côté des exogenèses qui s'imposent, y a-t-il pour vous des intertextes impossibles?

P. M. - Oui, bien sûr! De plusieurs espèces... Et eux aussi s'imposent dans leur impossibilité ! C'est même un aspect essentiel du phénomène : l'exogenèse par défaut. Les intertextes interdits sont en premier lieu ceux que tout le monde s'autorise : les exogenèses imitatives. Faulkner, par exemple, qui est l'un des plus séduisants, et donc l'un des plus dangereux intertextes. Tout le monde écrit en Faulkner. Jusqu'à présent, je crois que j'ai réussi à y échapper - sauf peut-être, par inadvertance, des fois? je me demande... Mais non, non, j'y suis arrivé ! J'ai des bouts de brouillons très faulknériens dans mes tiroirs, des fragments que je n'ai pas pu m'empêcher d'écrire en Faulkner : ils n'ont pas dépassé le stade du premier jet. Je m'en méfie terriblement parce que ça vient tout seul.

Par exemple, à un moment, j'avais commencé un récit qui s'intitulait «Vie de Jeannot Bonnet». C'était l'histoire d'un type mort en Corée. Il s'était engagé dans le corps expéditionnaire. Je l'avais connu juste avant, quand j'avais cinq ou six ans. Un jour, beaucoup plus tard, en lisant un bouquin d'histoire sur la guerre de Corée, je découvre qu'il avait été un véritable héros. Je me dis : il faut vraiment que j'écrive son histoire. J'étais enthousiaste. J'ai fait deux chapitres. C'était du pur Faulkner. C'était entièrement dicté par le rythme faulknérien, j' ai eu la sensation d'un artifice.
Ça s'est arrêté là. Il y a longtemps... c'était juste après Les Vies minuscules.

P.-M. B. - Faulkner et Céline : ce sont les deux intertextes impossibles, c'est bien cela?

P. M. - Oui, Céline c'est pareil, il est aussi dangereux, pour d'autres raisons. Mais là, je ne risque rien. Ce sont deux intertextes carrément impossibles parce qu'ils induisent chez tout le monde un tel mimétisme qu'il n'y a plus rien à en tirer. Ce sont des voies sans issue, des impasses mortelles : si vous entrez dans leur rythme, leur syntaxe et leur univers, vous êtes coincés, c'est fatal, vous n'en sortirez plus.

\section{Entendre des voix}

P.-M. B. - Donc, à l'exception de quelques emprunts appelés par la phrase qui s'écrit, l'exogenèse littéraire, pour vous, consiste moins à aller se saisir de tel ou tel joyau d'écriture, que de se laisser inspirer le désir d'écrire par la pulsation d'un intertexte, sans s'y assujettir. Il s'agit de faire corps avec la machine imaginaire et rédactionnelle d'un grand écrivain, pour donner à sa propre machine la force de démarrer puis la liberté de suivre ses propres rails?

P. M. - Oui, on pourrait dire ça comme ça... En fait, l'intertextualité est toujours là, en marge, à la marge de tout ce qui s'écrit; et l'exogenèse, la mobilisation effective de l'intertexte dans le mouvement de la rédaction, c'est une espèce de jeu de rôle, de dépersonnalisation ou de changement d'identité. On devient une sorte de canal par lequel passe le flux de l'intertexte, exactement comme on devient ce à travers quoi le texte s'écrit. Il s'agit de se laisser traverser. Cela peut aller jusqu'à une sorte de dédoublement de la personnalité : si je suis en train d'écrire quelque chose qui me fait penser que je suis Victor Hugo, il me viendra du Victor Hugo. Mais ce n'est pas une affaire d'intermittence, d'opportunités ou de moments, c'est un processus continu : c'est toujours là, en permanence, comme le lien d'Aragon avec Victor Hugo. Vous transformez l'intertexte, vous changez le sens et la forme de ce que disait l'autre, mais vous n'y échappez pas. Ce n'est pas intermittent, mais ce n'est pas non plus univoque. Ça peut être Hugo, et trois minutes plus tard Montaigne, ou Rimbaud, 
ou Flaubert, ou Kafka... Il s'agit d'être habité, mais pas par un seul fantôme. Si l'écriture est vraiment là, vous êtes habité par de multiples voix qui résonnent entre elles et en vous, qui se croisent dans votre oreille. J'entends positivement des voix... elles me parlent, elles se parlent, et autant que je peux, je les parle, mais avec mon langage.

P.-M. B. - Jeanne d'Arc revisitée par la polyphonie de Bakhtine! Si l'intertexte a pour vocation de devenir une chose si intérieure, ou plutôt s'il déjoue toute intériorité en faisant de l'écrivain son canal, sa simple médiation, est-ce que l'exogenèse ne devient pas indissociable, indistincte même de l'endogenèse?

$P . M$. - Ah oui, il y a toute une zone grise où ce n'est pas très facile de départager l'intérieur et l'extérieur. Mais d'un autre côté, non, on ne peut pas faire l'économie de l'opposition endo/exo parce qu'à côté de ces phénomènes, il y a aussi des cas, tout à fait précis et délibérés, où il s'agit bien d'exogenèse caractérisée, prédatrice, opportuniste. Tenez, par exemple, dans Les Onze, à un moment, je dis que Corentin, pendant la Terreur, avait une houppelande «couleur de fumée d'Enfer» : ça vient d'un texte de Jules Simon sur Michelet, un passage où il revoit Jules Michelet au petit matin, partant pour son cours au Collège, avec sa fameuse houppelande couleur de fumée d'enfer. Là, c'est de l'exo pure et simple : ce n'est pas une vision qui m'habite, ni un message des voix; c'est un truc que je découvre en faisant de la doc sur Michelet et qui me paraît si bon que je décide aussitôt de le transposer sur un autre personnage, Corentin : exactement comme la calotte de soie de Sainte-Beuve que j'ai collée sur la tête de Banville dans Rimbaud.

P.-M. B. - Déshabiller Michelet et Sainte-Beuve pour habiller Corentin et Banville!

P. M. - Ah oui! Les vêtements, il n'y a que ça de vrai. Ils ne font pas que le moine. Jamais je ne décris les traits de mes personnages, à l'exception d'Yvonne, bien sûr, dans La Grande Beune. En tout cas, dans Les Onze, la houppelande de Corentin, c'est de l'exo. D'ailleurs, la formule est en italiques... Mais, tout shakespearien qu'il paraisse, et précisément parce qu'il est shakespearien, il se peut que le «Où en est la nuit, Monsieur?» soit également de l'exo au sens plein du terme, parce que, pour moi, bien souvent, l'exogenèse la plus ponctuelle, répond aussi à un calcul de plus grande envergure : en l'occurrence, ici, à un contraste, à un partage de valeurs dans le grand équilibre des couleurs que je voulais construire. La deuxième partie des Onze devait être de coloration Macbeth, c'est-à-dire noire, tandis que la première devait être de coloration La Tempête, claire. En revanche si, oubliant l'interdit, j'écris : virgule, «la nuit», point, là ce n'est plus de l'exo, mais du flux d'écriture qui laisse agir le souffle de Flaubert dans ma phrase, c'est intérieur, c'est endo. Dans La Beune, il y a de l'intertexte savant, mais là c'est plutôt référentiel : la Préhistoire, Leroi-Gourhan, Bataille...

\section{La médiation référentielle des carnets}

P.-M. B. - ... Sans oublier, pour le deuxième volume de La Grande Beune, que tout le monde espère, tous les intertextes, ethnographiques et érotiques consignés dans vos notes, qui attendent de se métamorphoser en récit. Ça nous ramène à l'exogenèse des carnets, dont on avait commencé à parler dans un précédent entretien pour Genesis 10. Ce qui frappe dans ces notes préliminaires, où vous avez accumulé de si grands trésors de documentation, et tant de citations, c'est la disproportion et la dépense : l'extraordinaire dilatation du domaine de l'exogenèse préparatoire par rapport à ses retombées effectives dans la rédaction. Je repense à la métaphore du percolateur ${ }^{11}$ et de l'expresso à l'italienne : quelques gouttes de café intense pour l'énorme quantité d'arabica moulu que traverse un mince filet d'eau bouillante.

P. M. - Disproportion et dépense, ça se discute... parce que, en réalité, j'en profite! Je n'arrête pas de me faire des expressos italiens avec mes notes de carnets. Je les lis et les relis pour le plaisir, je les revisite si souvent que je finis par savoir par cœur ce qu'ils contiennent. Au total c'est un bon outil pour passer de l'exo à l'endo, pour acclimater l'exo aux

10. Pierre-Marc de Biasi, «Les Carnets inédits de La Grande Beune. Entretien avec Pierre Michon», Genesis, no 18, 2002, p. 117-127 en ligne sur persee.fr).

11. Pierre Michon. Retour aux origines, un film de Pierre-André Boutang et Pierre-Marc de Biasi. Production on line pour Arte, 2003, «Metropolis », no 443, samedi 6 septembre 2003. Script disponible sur pierre-marcdebiasi.com. 
besoins de la rédaction, pour le rendre capable d'informer l'écriture, notamment, dans tout ce qui relève des savoirs, de la pensée. Dans Les Onze, ce qui est dit sur les Lumières, sur le fait que Dieu s'est trouvé remplacé par le Texte, etc., tout cela finit par se ramasser dans la formule «Dieu changeait de nid». Cette expression, pour le coup, est bien de moi, mais ce qu'elle dit, et toutes les idées qui soutiennent le passage, relèvent strictement de l'exogenèse : c'est du Benichou monté à l'épique, c'est de l'exo pur, ni plus ni moins.

Les idées, ce n'est pas mon fort : quand c'est au niveau de la pensée, des savoirs et des concepts, c'est toujours de l'exo. Moi-même, je déblatère des phrases, je rumine de l'alexandrin, je me délecte de citations... mais je ne pense pas. Il y a de l'ivresse à faire de la doc encyclopédique, mais il y a surtout pour moi l'impérieuse nécessité de consigner dans mes carnets tout ce dont j' aurai besoin pour ne pas dire trop de bêtises sur les sujets - techniques, historiques, référentiels, etc. - où je sais que le texte risque de m'entraîner. Pour les connaissances, les théories, les informations techniques qui en elles-mêmes n'ont pas grand-chose à voir avec la littérature, l'exo est vraiment extérieure; et le carnet est une façon de se les approprier, de les recadrer et de les rassembler pour se les rendre disponibles. C'est de l'info qui peut avoir été réunie pour un projet de rédaction précis; il peut y voir des citations, littéraires ou non, des notes d'idées, même des amorces de textes endogénétiques, mais ça peut aussi contenir des choses très digressives par rapport au projet... Je m'en sers de manière plus ou moins aléatoire pendant la rédaction.

\section{L'impulsion rythmique}

P.-M. B. - Mais il y a pour vous une différence de nature entre la note informative, la citation ou le détail étrange, que le carnet consigne par provision pour nourrir la narration, et ce bain exogénétique purement littéraire dans lequel il s'agit d'entrer pour trouver le tempo de l'endogenèse, pour activer l'écriture, pour l'invoquer en quelque sorte?

P. M. - Oui, quand on entre à proprement parler dans l'espace littéraire, les choses commencent à devenir paradoxales : l'exogenèse que portent les textes littéraires ne peut pas se distinguer de l'intériorité endogénétique qu'ils mobilisent et qui les mobilise. C'est un échange, une transfusion réciproque. Dès qu'il y a frisson littéraire, c'est l'ensemble des textes littéraires qui viennent se remémorer en moi pour m'accompagner dans l'écriture : tout un monde de rythmes, de mots et d'images qui existe assurément hors de moi dans des livres - et en cela qui relève bien de l'exogène - mais un monde qui me traverse et qui me constitue dans ma capacité à écrire - et en cela que je ressens comme endogène -, quand par bonheur je me redécouvre cette capacité.

P.-M. B. - Donc, dans l'espace littéraire proprement dit, pas de frontière précise entre exo et endo?

$P . M$. - Non, pas de frontière : quand il y a la grande vague qui vous porte, c'est de l'endo. Ça n'empêche pas qu'il puisse y avoir, localement, retour de l'exo, en tant que tel, dans certains gestes plus concertés de la rédaction. Il y a exo quand il y a préméditation et crime. Mais ça reste exceptionnel.

P.-M. B. - Et il ne pourrait pas y avoir exogenèse rédactionnelle à une autre échelle? En termes de structure, de forme ou de mouvements du récit?

P. $M$. - Je ne crois pas trop. Mais peut-être. Certains modèles sont plus efficaces que d'autres. Par exemple, il y a un procédé utilisé par Hugo, et par bien d'autres également, qui consiste à commencer le récit, ou même chaque chapitre, chaque début de mouvement par une toute petite phrase. Une phrase d'incipit brève pour faire démarrer le texte, et puis on embraye. Rien n'est meilleur pour la santé narrative. Hugo ne rate pas une occasion. Les auteurs de polar ne s'en privent pas. Ça m'arrive aussi. Mais au fond, ce n'est pas exactement de l'exogenèse, c'est une option de forme, un procédé.

Quant à chercher des modèles pour préfigurer l'architecture globale d'un texte, je n'y crois pas beaucoup, et pour ce qui me concerne, ça n'aurait pas de sens : je ne travaille pas avec des plans, j'écris à la va comme je te pousse, c'est la rédaction qui crée la forme, vous le savez bien! Non, par exemple, si j'avais à écrire quelque chose, là, tout de suite, il faudrait juste que j'ai un tempo dans la tête, une scansion, un rythme : par exemple deux vers de refrain pris à la «Chanson des pirates », toujours de ce satané Hugo !

Dans la galère capitane

Nous étions quatre-vingts rameurs. 
Voilà... juste deux octosyllabes, une galère, des rameurs, et ça suffit : ça induit une pulsation pour rythmer une phrase, quel que soit son contenu; ça peut aussi suggérer une foule d'images, une thématique, un désir d'histoire, une histoire de navigation, de forçats... Est-ce de l'exogenèse?

Mais puisqu'on parle de rameurs, ça me fait penser à un autre cas d'intertextualité qui renvoie bien à un fait d'exogenèse. Dans mon texte sur Goya, il y a une histoire de galériens : je dis que peindre c'est comme ramer, comme être forçat sur une galère, etc. (j'aime bien les galères). Ça vient de Balzac. Et je m'en suis aussi servi dans Les Vies minuscules. C'est le moment des Illusions perdues où Vautrin dit à Lucien de Rubempré : mon petit gars, ou bien tu me suis, tu écris tes petits torchons et je te les place, ou bien tu vas écrire sur l'eau avec des plumes de quinze pieds ! J'adore le face-àface Vautrin/Rubempré. Le personnage de Jeanjean dans La Grande Beune a quelque chose de Vautrin. Quand j'aime, je ne fais pas la fine bouche, j'y vais à la hache. Donc l'intertexte Balzac traverse mes récits. Mais c'est le cas également pour l'intertexte Dumas : La Grande Beune est pleine d'emprunts aux Trois Mousquetaires. Oui, entre autres, pour la figure de Milady : «Où vais-je mourir? dit-elle. - Sur l'autre rive12.»

À propos, vous savez? Je l'ai fait, le deuxième volume de La Grande Beune! Il est terminé. Mais je ne le donne pas encore... et je ne vous en dis pas plus. Mais c'est bien sur l'autre rive que ça se passe, comme dans Les Trois Mousquetaires!

\section{Exogenèse contemporaine}

P.-M.B. - Le deuxième volume de La Grande Beune est écrit! Quelle nouvelle! J'imagine que ça ne portera plus le titre prévu dans les carnets : L'Origine du monde. On en parlait en 2002. Nous voilà presque vingt ans après, comme dans la suite des Trois Mousquetaires!... Mais, puisque vous ne voulez pas en dire plus, revenons à votre nouveau roman. C'est l'histoire d'un grand écrivain de notre temps avec, dites-vous, de multiples jeux d'exogenèse. Ils pourraient être l'occasion de mettre en scène l'intertexte contemporain, et peut-être même de le bousculer? Est-ce le cas?

$P$. $M$. - Le héros est un écrivain que je dirais de type Houellebecquo-borgésien. L'intertexte borgésien, je le porte avec moi depuis toujours. Pour le reste, non, je ne cherche pas à régler le moindre compte avec l'actualité littéraire. Il ne s'agit pas pour moi d'écrire contre qui que ce soit. Écrire autrement, ça oui : pas d'hostilité, un peu d'altérité, de l'écart. J'ai des stratégies défensives en me tenant à l'abri des modèles qui cannibalisent, style Faulkner : mais je n'ai pas de stratégie offensive. D'ailleurs, rien ne me révolte dans la littérature d'aujourd'hui. J'ai plutôt l'impression qu'il y a toujours quelque chose de bon à y puiser, ici ou là : d'abord comme lecteur (parce que je ne suis pas difficile) et ensuite comme écrivain (parce que je suis du genre prédateur)... il y a plein de trucs intéressants dont je me sers dans les bouquins qui paraissent, dans les séries, dans les films, ceux d'aujourd'hui et les classiques. Pour mon livre en cours, je viens de faucher une citation de Sérotonine de Houellebecq et une réplique d'Orson Welles, au début de La Dame de Shanghai. Et là je me demande si je ne vais pas piquer une autre réplique que je viens de trouver, pas plus tard qu'hier, dans un polar, mais qui doit venir d'un western, j'en suis quasiment certain : «Si vous voulez amuser Dieu, dites-lui que vous avez des projets... » Pas mal, non ? Donc n'importe quoi peut venir dans mes textes. Dans le Watteau je m'étais amusé à faire du Duras : j'avais repris l'exclamation du Vice-Consul «Ana-Maria Guardi!», pour écrire «MarieLouise Gersaint»13. Je ne sais pas si la femme de Gersaint s'appelait Marie-Louise, mais je voulais Marie pour rappeler Maria. Là, je le reconnais, il faut vraiment le savoir, c'est un emprunt presque secret, mais c'est bien Duras qui travaille dans ma phrase...

P.-M. B. - En dehors de Houellebecq, comment vous situez-vous par rapport à l'état présent de l'intertextualité littéraire?

P. M. - Je ne me situe pas... Le grand intertexte pour les contemporains, c'est les Anglo-Saxons : Faulkner, John

12. C'est Milady qui parle avant son exécution : «Où vais-je mourir? dit-elle. - Sur l'autre rive, répondit le bourreau. Alors il la fit entrer dans la barque, et, comme il allait y mettre le pied, Athos lui remit une somme d'argent. - Tenez, dit-il, voici le prix de l'exécution [...]» Alexandre Dumas, Les Trois Mousquetaires, Ch. LXVII, «L'exécution».

13. Pierre Michon, Maîtres et Serviteurs, «Je veux me divertir», Verdier, 1990 , p. 83 : «Et plus tard, d'une voix forte, comme un cri neutre "MarieLouise Gersaint !" ». 
Fante, Bukowski, Tom Wolfe, Bret Easton Ellis, etc. J'ai lu, mais ce que je fais n'a pas grand rapport avec ce genre de romans, je ne suis pas dans cette zone d'influence, ça ne me concerne pas réellement. Il faut dire que c'est aussi une histoire de chronologie personnelle : tout ce qui marche bien pour moi, c'est ce que j'ai stocké entre dix-sept et vingt-cinq ans, dans les années 1960-1970. Les Américains, je les ai lus, mais plus tardivement, j'étais déjà engagé dans l'écriture. En dehors de Faulkner, ils ne sont pas dans mon champ de vision.

\section{Intertexte artistique}

P.-M. B. - Au sens large, le processus intertextuel ne se limite pas à l'univers des traces écrites. L'intermédialité qui joue un rôle si important dans votre travail oblige à revenir sur le rôle des images, des cuvres d'art et de l'intertexte artistique. Que dire, par exemple, de cette inter-iconicité qui relève plus de l'histoire de l'art que de la critique littéraire, mais qui semble travailler en profondeur votre imaginaire d'écrivain et agir sur votre travail?

$P . M$. - C'est-à-dire qu'en effet, quand je parle de tableaux, j'ai souvent lu beaucoup de textes sur ces tableaux. À côté des œuvres et leurs représentations, j'ai en tête toute une petite bibliothèque sur ce que je vois. C'est une affaire de surchauffe imaginaire. Au départ, il y a un tableau qui vous chauffe l'imagination, puis derrière lui, il y a tout le baratin des historiens d'art que vous lisez, tous les commentaires écrits sur ce tableau, qui le portent au rouge, et enfin, il y a ce que vous allez en dire, et là, il faut que ça passe à l'incandescence absolue, que ça le porte au blanc. En fait, il s'agit de grimper une pente d'autosuggestion faite de représentations et d'interprétations, de citations et de visions de l'œuvre, qui font monter de plus en plus haut la colonne de mercure du thermomètre. C'est le même processus en littérature avec les textes et les commentaires, et on peut y voir un danger. Nizan par exemple insistait beaucoup là-dessus : Méfiez-vous terriblement des textes qui portent l'œuvre littéraire à la température d'un dieu ! Il n'aimait pas ça du tout. Moi si ! J'aime le chaudron et la fournaise : plus il y a de textes et d'images pour faire monter la chaleur et atteindre le point de fusion, et mieux je me sens : vous malaxez la matière incandescente des textes et des images, puis vous trouvez la formulation! ou bien vous ne la trouvez pas, et vous faites comme si vous l'aviez trouvée!

P.-M. B. - On n'est pas loin de la fièvre comme signe de «haute santé» dans le Van Gogh d'Artaud.

P. M. - Oui ! et justement, pour la Vie de Joseph Roulin, c'était le risque majeur. Pour ce récit, il fallait que j'évite avec le plus grand soin tout artaldisme. Et, de fait, il n'y en a pas... à une petite exception près : l' «édredon rose crevette ${ }^{14}$ » qui est quand même dans Les Vies minuscules.

P.-M. B. - Cette procédure d'exclusion est aussi une forme d'intertextualité négative, mais ici, par rejet circonstancié : pour ce texte, tout sauf ceci ou cela! Faulkner ou Céline relèvent de l'interdit permanent, du bannissement à perpétuité; pour d'autres intertextes, c'est l'interdiction de séjour. La peine est plus légère : elle est prononcée à titre provisoire, en raison du sujet traité, pour la durée de la rédaction.

P. M. - Absolument : surtout pas cela maintenant ici! Le risque est trop grand. Et c'est pour ça que mon Van Gogh, dans le fond, a fini par prendre des teintes à la Corot plus qu'une coloration Van Gogh : c'était l'autre risque, mais il n'y avait pas le choix. Plutôt Alphonse Daudet qu'Antonin Artaud! Il était crucial de se démarquer, quitte à perdre en intensité du côté de la peinture et du mythe. Dans l'exogenèse négative, dans cette injonction à ne pas toucher à tel ou tel intertexte, il s'agit toujours plus ou moins d'échapper à du convenu; avec le Van Gogh d'Artaud, l'exigence était encore plus impérieuse : il s'agissait d'échapper à du non-convenu déjà réalisé et réussi au plus haut degré.

14. «Mais comment faire comprendre à un savant qu'il y a quelque chose de définitivement déréglé dans le calcul différentiel, la théorie des quanta, ou les obscènes et si niaisement liturgiques ordalies de la précession des équinoxes, - de par cet édredon rose crevette que Van Gogh fait si doucement mousser à une place élue de son lit, de par la petite insurrection vert Véronèse, azur trempé de cette barque devant laquelle une blanchisseuse d'Auvers-sur-Oise se relève de travailler, de par aussi ce soleil vissé derrière l'angle gris du clocher du village, en pointe, là-bas, au fond de cette masse énorme de terre qui, au premier plan de la musique, cherche la vague où se congeler», Antonin Artaud, Van Gogh ou le suicidé de la société, 1947. 


\section{Prendre, ne jamais imiter}

P.-M. B. - En quoi l'exogenèse reformule-t-elle, selon vous, la question de l'imitation? Si l'écrivain digne de ce nom est celui qui s'interdit d'imiter, quelle relation peut-il instaurer à l'égard des intertextes qu'il se donne comme modèle, ou dont il se sert dans son écriture?

P. M. - L'écrivain est un prédateur. Il se donne le droit de prendre. Comme disait Roth, je crois bien que c'est lui qui disait ça : «Prendre! ne jamais imiter. » Parce que prendre, c'est transformer, mettre dans une autre machine, greffer sur un autre tissu, un autre corps. Si la greffe ne marche pas, s'il y a rejet, c'est qu'il y a incompatibilité. Et là il n'y a aucune différence entre endogenèse et exogenèse : le principe exo, c'est prendre, mais il faut aussi que ça prenne dans l'endogenèse. Au sens de la mayonnaise ou du béton. Et c'est instantané. Ou bien tout converge dans la rédaction pour que le fil soit pris dans la trame, ou bien tout s'effiloche et il faut y renoncer. Mais pour que ça prenne, il faut en réalité une conversion immédiate de la matière intertextuelle. Mon projet sur La Vie de Jeannot Bonnet a tourné court parce que l'écriture était restée bloquée sur le modèle faulknérien, je n'étais pas parvenu à convertir son impulsion en énergie propre. Là, c'est bien à cause de l'intertextualité que le texte n'a pas pu aboutir. C'est une affaire de voix. S'il y a emprunt à un intertexte, il faut que ce soit repensé et rebrassé de l'intérieur, et tout de suite porté par une force plus grande que celle de la voix qui vous parle. La voix de Faulkner, je l'ai toujours dans l'oreille, mais je lui interdis de se substituer à la mienne. Il vaut beaucoup mieux, d'ailleurs, qu'il n'y ait pas qu'une seule voix. C'est bien qu'il y en ait plusieurs, qu'elles se promènent en tournant autour de votre phrase, mais à la manière des bonnes sœurs en patin à roulettes de Fellini Roma : en passant et en repassant, à condition qu'elles finissent par prendre la tangente et disparaître...

Voilà pourquoi les carnets ne sont pas seulement des outils d'information préparatoire, mais constituent aussi pour moi un dispositif de sécurité : en préparant un projet, je fais provision des intertextes de toutes natures qui me seront utiles, sans négliger le superflu ni la divagation, mais surtout je diversifie les voix qui pourront, le moment venu, parler à mon oreille. C'est un dispositif d'écoute polyphonique. De plus, le carnet a ceci de particulier qu'il autorise, par sa forme, les usages les plus divers en matière d'écoute. On peut le consulter pour aller y chercher ce dont on a besoin en essayant de retrouver la page où ça se trouve. Mais on peut aussi le compulser en le feuilletant à l'aveuglette, pour tomber sur de l'inattendu.

P.-M.B. - L'intertextualité aléatoire, une sorte de tombola exogénétique! Et pour le livre que vous êtes en train d'écrire, vous avez ouvert beaucoup de carnets avant de vous mettre à l'écriture? Combien?

P. M. - Beaucoup trop! dans les 25, 26, je crois... Et c'est peut-être pour ça que je suis resté embourbé dans ce projet depuis si longtemps. Trop de notes préparatoires peut nuire à une rédaction. On sait trop de choses, ça fait hésiter. Ça fait trop réfléchir. Alors, j'utilise les carnets le plus légèrement possible, parfois en les ouvrant au hasard, à n'importe quelle page, pour l'effet d'aubaine : «Ah ! oui ! voilà un détail, un rythme, une image, une formulation... qui irait bien avec ce que j'ai écrit tout à l'heure ! »C'est la roulette, une sorte de pari sur le hasard objectif, et quelquefois ça fonctionne ! 36 fois la mise! Mais pour que ça fonctionne, il faut que ce ne soit pas seulement un jeu intellectuel. Ça ne peut pas être trop concerté; ni préparé, sinon ça ne marche pas. Il faut vraiment pouvoir se laisser surprendre. Il faut que ça ait lieu dans le feu de l'écriture, avec l'émerveillement de la trouvaille, la joie de la découverte qui se traduit immédiatement en phrases.

Dans mon projet actuel, comme le narrateur est un écrivain plutôt sûr de lui, ironique mais très savant, qui gère toutes les postures auctoriales, et qui est saturé d'intertextes, la roulette peut marcher à fond : faites vos jeux, rien ne va plus, et hop! une formule de Hugo, un rythme de Borges, une apostrophe d'Artaud... Le personnage ne le dit pas, mais ça se dit à travers lui, et les intertextes défilent.

P.-M. B. - Si bien que finalement, le jeu intertextuel, c'est le héros lui-même qui se met à l'incarner. Plus fort que la résurrection de Michelet dans Les Onze! En s'énonçant à travers le personnage de l'écrivain, les figures de l'exogenèse prennent corps et voix, et la fiction intertextuelle devient performative : par l'énonciation elle accomplit ce qui est dit.

P. M. - Oui, c'est une des singularités du récit : dans cette fiction, ce sont les figures de l'intertexte qui tiennent les commandes de l'énonciation. 


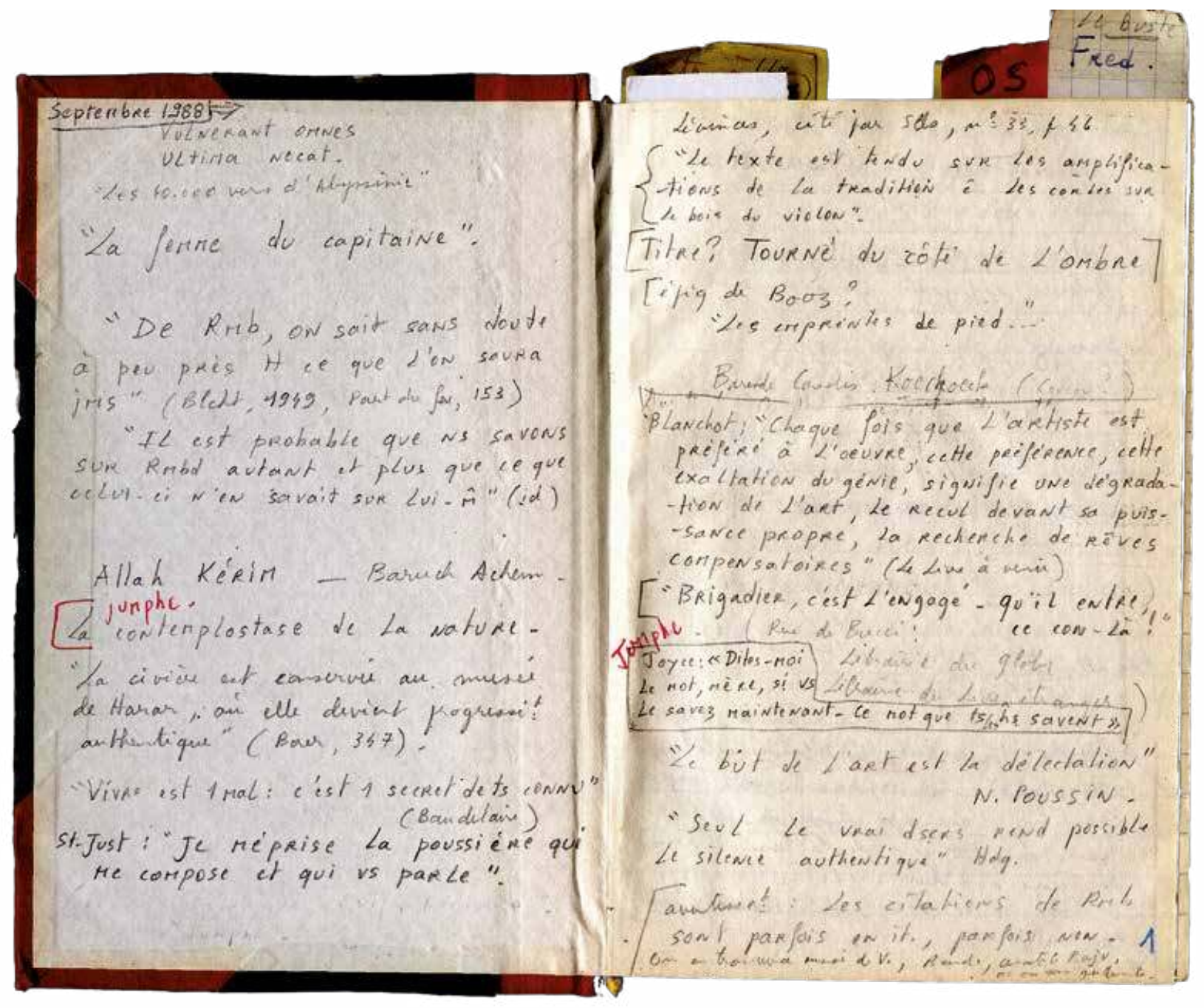

Carnet de Pierre Michon ayant servi à l'écriture de Rimbaud le fils paru en 1991 chez Gallimard. 